meteorological factors and $\mathrm{NO}_{2}$ were controlled, an increase in 10 microg/m (3) in PM (2.5) was associated with EHVs for cerebral haemorrhage with ORs of 1.168 (95\% CI 1.000, 1.363). But there was no relationship between the daily average PM (10) concentration and EHVs. The association between PM2.5 and EHVs for hypertension was significantly modified when the mass was high in $\mathrm{Ni}$ or $\mathrm{V}$.

Conclusions Elevated urban gaseous air pollution was associated with increased EHVs for cerebral haemorrhage in Changsha, China. PM (2.5) mass higher in Ni and V significantly increased its effect on EHVs for hypertension.

\section{GW23-e2321 AMBIENT FINE PARTICLE METAL COMPONENTS AND EMERGENCY HOSPITAL VISITS FOR HYPERTENSION IN CHANGSHA, CHINA: A TIME-STRATIFIED CASE- CROSSOVER STUDY}

doi:10.1136/heartjnl-2012-302920d.24

${ }^{1}$ Zhi-jun Huang, ${ }^{1}$ Hong Yuan, ${ }^{1} Y u$-qing Zhou, ${ }^{2}$ Oi-hong Deng, ${ }^{2}$ Chan Lu, ${ }^{1}$ Ming Cai, ${ }^{3}$ Hong Yuan. ${ }^{1}$ The Third Xiangya Hospital of Central South University; ${ }^{2}$ The Central South University; ${ }^{3}$ The Third Xiangya Hospital of Central South University

Objectives To explore the risk effect of fine particle and metal components on the emergency hospital visits (EHVs) for hypertension in Changsha, China.

Methods We gathered data on daily EHVs for hypertension from the third Xiangya hospital of central south university, collected metal components concentration of fine particulate matter less than $2.5 \mu \mathrm{m}$ in aerodynamic diameter (PM (2.5)), particulate matter less than 10 microm in aerodynamic diameter (PM (10)), suphfur dioxide, and nitrogen dioxide in Changsha, China during June and October in 2009. A time-stratified case-crossover design with distributed lag model was used to evaluate associations between ambient air pollutants and hypertension. Daily mean temperature, atmospheric pressure and wind velocity were controlled in all models.

Results There were 1027 EHVs for hypertension during the study period. After daily mean temperature, atmospheric pressure, wind velocity, $\mathrm{NO}_{2}$ and $\mathrm{SO}_{2}$ were controlled in models, an increase in $10 \mu \mathrm{g} / \mathrm{m}$ (3) in PM (2.5) was associated with EHVs for cerebral haemorrhage with ORs of 1.177 (95\% CI 1.006, 1.376); after 